\title{
A New Spatial Path Persistency Model for TOA-Based Indoor Geolocation
}

\author{
Ferit Ozan Akgul, Member, IEEE, and Kaveh Pahlavan, Fellow, IEEE
}

\begin{abstract}
Analysis of the persistency and birth/death of multipath components of the channel impulse response is essential for performance evaluation of indoor geolocation algorithms using indirect paths for ranging. The use of indirect paths via multipath diversity is particularly important for situations where the direct path gets blocked. In this letter, we propose a statistical model for the spatial behavior of the persistency of multipath components as a mobile moves inside a building. Based on empirical data from a measurement calibrated ray tracing algorithm we show that the persistency in a typical building is modeled by log-logistic distribution rather than commonly assumed exponential distribution and path birth can be modeled as a Poisson distribution.
\end{abstract}

Index Terms-Path persistency, path lifetime, spatial path behavior, TOA-based indoor geolocation.

\section{INTRODUCTION}

$\mathbf{I}$ NDOOR wireless propagation has been extensively studied primarily for communications applications for both static [1], [2] and dynamic cases [3], [4]. However, different factors need to be considered when modeling is needed for indoor geolocation applications.

Indoor geolocation using RF signals has many challenges since indoor environment has rich and densely spaced multipath components (MPCs). For time-of-arrival (TOA) based geolocation applications, TOA of the direct path (DP) is an important feature. However, due to certain obstructions between the transmitter and the receiver, such as metallic objects, the power of the DP and the nearby MPCs may be reduced below detection threshold. This leads to a condition called undetected-direct-path (UDP) causing large ranging errors [5]. The modeling of UDP ranging errors has been studied in [6].

Under UDP conditions, spatial behavior of the MPCs caused by the dynamic movement of the receiver is important since they can be utilized for multipath diversity [5], [7]. When performance evaluation of algorithms using multipath diversity is needed, two metrics gain importance. The first and most important of these metrics is the spatial path persistency, which is the lifetime of a path as the receiver is moving at a given velocity $(v)$. The second is the birth rate at which paths appear during this motion. Path death is dictated by the path lifetime. The available path lifetime models in the literature are [3], [4] which were developed for communication applications. In [3] path birth/death is modeled by a marked Poisson process and hence path lifetime is exponentially distributed. The study in

Manuscript received November 16, 2008. The associate editor coordinating the review of this letter and approving it for publication was C. Assi.

The authors are with the Center for Wireless Information Network Studies, Worcester Polytechnic Institute, Worcester, MA, 01609 USA (e-mail: ferit@wpi.edu).

Digital Object Identifier 10.1109/LCOMM.2009.081932
[4] follows a different approach and use multi-state Markov model to describe path birth/death and exponential distribution for path lifetime.

In this letter, we present a new model for the persistency and birth rate of the MPCs for indoor geolocation. We examine exponential, Weibull and log-logistic distributions for path persistency and show that exponential distribution suggested in [3] and [4] provides an optimistic model while log-logistic persistency model together with Poisson birth rate provide the best conformance to empirical results. We have obtained the parameters of the models by a measurement-calibrated raytracing (RT) tool on the 3rd floor of the Atwater Kent (AK) Labs at Worcester Polytechnic Institute (WPI).

\section{Dynamic Multipath Model Based on GEOMETRICAL INTERPRETATION}

In order to describe the evolution of multipath features, we will regard each MPC as an infinite bandwidth optical ray whose behavior is dictated by major indoor propagation mechanisms, namely reflection and transmission. This modeling scheme was first proposed by Turin [8] and is well suited for RF propagation in multipath-rich indoor environments.

Since we will be considering the dynamic case for the receiver, we can express the $x$ dependent spatial channel by

$$
h(x ; t, \tau)=\sum_{i=1}^{L_{p}} \beta_{i}(x, t) e^{j \phi_{i}(x, t)} \delta\left(t-\tau_{i}(x)\right)
$$

where $L_{p}$ is the number of MPCs, $\beta_{i}$ and $\phi_{i}$ represent the amplitude and phase of the $i^{\text {th }}$ path, and $\tau_{i}$ is the propagation delay of the $i^{\text {th }}$ path. Based on (1), we can easily see that one of the unique features of a single MPC is its TOA which is the primary feature for TOA-based indoor geolocation.

As the receiver is moving steadily on a given route its TOA will exhibit differential changes as long as there is no major change in the propagation scenario. Propagation scenario is tightly related to the internal structure of the indoor environment, and is primarily dependent on the reflection mechanism. We can express the reflection mechanism as a vector whose elements are an ordered set of surface/wall indices. For instance, a path that goes through 2 reflections at walls $w_{1}$ and $w_{2}$ respectively would have a reflection mechanism $\left[w_{1} w_{2}\right]$. Changing the order of reflections would create a different reflection mechanism hence a different propagation scenario. We will use the notation $P_{i, x}=\left[w_{1} w_{2} \ldots w_{n}\right]$ to denote the propagation scenario of the $i$ th MPC at position $x$ after getting reflected at the walls $w_{1}, w_{2} \ldots w_{n}$.

Persistency of a certain MPC is the time interval expressed in number of discrete time steps (or equivalently the distance that the receiver moves given its speed) in which the propagation scenario stays intact. In other words, having 


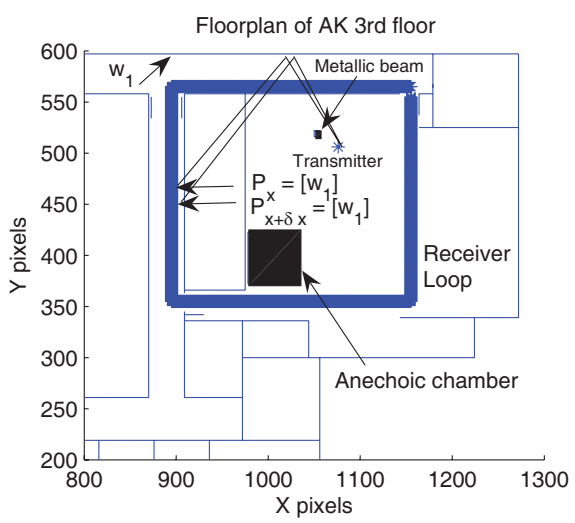

Fig. 1. Test loop on AK 3rd floor, showing a persisting path in interval $\Delta X$.

$P_{i, x}=\left[w_{1} w_{2} \ldots w_{n}\right]$ at position $x$ and $P_{i, x+\delta x}=\left[w_{1} w_{2} \ldots w_{n}\right]$ at position $x+\delta x$ would indicate a persistency of $\delta x$ for the $i$ th path. This is also depicted in Figure 1 for an MPC with only one reflection from the top wall, $w_{1}$.

\section{PATH BIRTh RATE}

As the receiver moves along a given route, many paths will appear and disappear. Path appearance is the formation of a new reflection mechanism so that an MPC can reach the receiver by this propagation scenario. Path disappearance, on the other hand, is the loss of a reflection mechanism since an MPC cannot reach the receiver via the same propagation scenario anymore. The disappearance or "death" of paths will be governed by the path persistency since a path will be assumed dead at the end of its persistency. However, we need to have a certain model for the generation or "birth" of new paths. The mechanism by which these paths are born has been studied through various models in the literature [3], [4] as mentioned before. Based on our observations using the RT simulations, we propose a Poisson birth model for the number of paths that are born. One previous study [2] proposed a Poisson model for the arrival of paths in the static case. In that study, experiments and simulations were performed at predefined transmitter or receiver locations and a number of channel impulse responses (CIRs) were collected. Hence these models are actually valid for the $\tau$ axis. Our model is defined in the spatial dimension, $x$, describing the spatial evolution of paths as the receiver moves. The model can be given as

$$
p\left(N(\Delta X)=n_{b}\right)=\frac{\left(\mu_{B} \Delta X\right)^{n_{b}}}{n_{b} !} e^{-\mu_{B} \Delta X}
$$

where $\mu_{B}$ is the birth rate, $n_{b}$ is the number of births in a given spatial interval, $\Delta X$. The Poisson modeling approach that we have adopted is actually based on a Bernoulli trial in which we assume either a "zero" or a "single" birth in an interval small enough to make our assumption valid. In this small interval, $\Delta x$, we assign a probability of $p_{0}$ for a nobirth case and a $p_{1}$ for a single birth. Our choice of the Poisson model stems from the fact that this simple yet powerful model can describe multiple stochastic occurrences of certain events in a given time. Hence the probability of occurrence of $n$ paths in a given interval $\Delta X=m \Delta x$ can be computed as

$$
p_{n}=\left(\begin{array}{c}
m \\
n
\end{array}\right) p_{1}^{n} p_{0}^{m-n}
$$

When $m$ tends to a large number and $p_{1}$ to a small number, the overall probability can be approximated by the Poisson random variable with the parameter $\mu_{B}=m p_{1}$.

\section{PAth Persistency}

The persistency of multipath components has not been studied extensively in the literature. The studies in [3] and in [4] are some previous and early works that model the duration for which multipath components stay active. Authors in [3] propose a Poisson birth/death process for multipath duration, hence the lifetime is modeled as an exponential random variable. The study in [4] similarly propose an exponential path lifespan based on experimental data obtained with a system bandwidth of $120 \mathrm{MHz}$. The exponential model with parameter $\lambda$ is given as

$$
f_{x_{P}}(x)=\lambda e^{-\lambda x}
$$

Although an exponential form is also a valid approach for modeling persistency, it is not adequate [9], [10]. Hence other modeling schemes need to be considered. One such method is the Weibull modeling approach written as

$$
f_{x_{P}}(x)=(b / a)(x / a)^{(b-1)} e^{-(x / a)^{b}}
$$

where $a, b$ are the scale and shape parameters respectively. However, based on our observations from the RT simulations, Weibull scheme would still not be able to provide an accurate representation of lifetime data. Hence, we are proposing loglogistic distribution for the persistency of a MPC. The use of log-logistic has been considered in other disciplines [11] as a statistical lifetime model. The log-logistic model is written as

$$
f_{x_{P}}(x)=\frac{e^{\frac{\ln (x)-\mu}{\sigma}}}{\sigma x\left[1+e^{\frac{\ln (x)-\mu}{\sigma}}\right]^{2}}
$$

where $\mu, \sigma$ are the scale and shape parameters, respectively, and $x$ is the distance receiver moves. Equivalently path persistency can be interpreted as the time duration, $t_{P}$; it persists through the relation $t_{P}=x_{P} / v$.

Among the three modeling schemes, namely exponential, Weibull and log-logistic, our observations indicate a better conformance to the log-logistic method. Since path persistency is directly related to the propagation scenario, the distribution of the interior wall lengths of the building has a direct impact on the distribution of path persistency. The log-logistic model can describe longer path persistencies that are produced by longer walls such as corridors better. The Exponential and Weibull models decay too quickly to describe this behavior.

Thus the complete spatial path behavior is modeled by the combination of the log-logistic path persistency and the Poisson birth model. Results in terms of cumulative hazard function and a two-sample Kolmogorov-Smirnov (KS) test will be presented next for a quantitative comparison of the aforementioned models and the suitability of our proposed model. 


\section{VERIFICATION OF THE MOdel BASED ON RT SiMULATIONS}

For verification, we utilized a calibrated RT tool [12], and developed realistic indoor floorplans of the AK Labs at WPI. Ray tracing methods have proven to be efficient for most studies [13]. The setup for the RT platform is shown in Figure 1. The choice of the receiver loop also presents a diversified $\mathrm{RF}$ environment with mixed detected direct path (DDP)/UDP conditions due to both macro and micro metallic obstructions including the anechoic RF chamber and the metallic vertical beam. Receiver motion is simulated by obtaining consecutive CIR responses at locations $5 \mathrm{~cm}$ apart from each other on the loop on the 3rd floor of AK. Hence the CIR results have been obtained with a spatial resolution of $5 \mathrm{~cm}$ corresponding to the floorplan scale of 20 pixels $/ \mathrm{m}$. This spacing also corresponds to the $\Delta X$ as described in the path birth model.

For the modeling of path birth, we have two free variables $p_{1}$ and $m$. By assigning a small probability value to $p_{1}$ we can obtain all the other related parameters. Specific to our case we have chosen $p_{1}=0.01$. Based on the RT simulation results, $\mu_{B}$ is calculated as 17 and thus $m=1700$. Hence $\Delta x=\Delta X / m=0.0029 \mathrm{~cm}$. Figure 2(a) shows the CDF for net path birth/death using the combined Poisson path birth and log-logistic path persistency model.

A two sample Kolmogorov-Smirnov (KS) test is used to test the suitability of the selected model. The $D$ statistic, given as:

$$
D=\sup _{x}|F(x)-S(x)|
$$

where $F(x)$ denotes the fitted distribution with estimated parameters and $\mathrm{S}(\mathrm{x})$ denotes the experimental data, can be interpreted as the greatest vertical distance between these two distributions and is a measure of how closely the proposed model describes empirical results. A smaller $D$ indicates a better overall fit. Comparison of this value against a tabulated critical value determines if the proposed (hypothesized) model can be accepted at 5\% significance level, which is a common value used in statistical tests. Based on this test and at 5\% significance, our proposed Poisson birth model is acceptable.

In order to obtain the persistency of a single path, a path tracking algorithm is employed. A version of this algorithm is discussed in [14]. For the comparison of persistency results, we have chosen the cumulative hazard function which is a common method used in survival analysis [10]. The cumulative hazard function can be written as:

$$
H(x)=-\log S(x)
$$

where $S(x)$ is the survivor function expressed as:

$$
S(x)=\operatorname{Pr}\left(x_{P} \geq x\right)
$$

which gives the probability that path persistency, $x_{P}$, is greater than $x$. As we can see from Figure 2(b), log-logistic modeling for path persistency is a more accurate approach following the cumulative hazard of the empirical results more closely. In this graph, $x$ axis is the lifetime in meters and $y$ axis is the $H(x)$. The KS results for the models based on $D$ statistic are also given in Table I for a quantitative comparison.
TABLE I

\begin{tabular}{ccc}
\hline Persistency Models & $D$ & Model Parameters \\
\hline Exponential & 0.1693 & $\lambda=1.1925$ \\
Weibull & 0.1342 & $a=0.7655, b=0.8703$ \\
Log-logistic & 0.0801 & $\mu=-0.8645, \sigma=0.5851$ \\
\hline
\end{tabular}

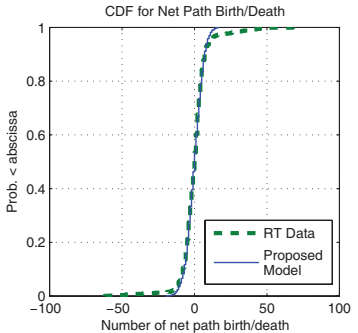

(a)

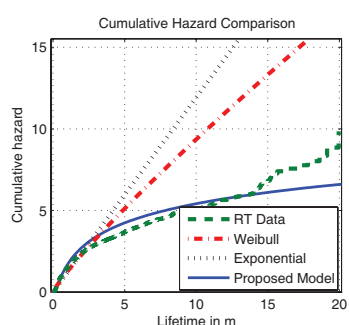

(b)
Fig. 2. (a) CDF for net path birth/death - (b) Cumulative hazard comparison for path persistency.

\section{CONCLUSION}

In this letter, we have introduced a combined multipath persistency and birth model in a dynamic scenario. The KS test yields the minimum $D$ value for the log-logistic model when compared to Weibull and exponential for path persistency indicating a better overall conformance to this model. The results of the calibrated RT tool show a good agreement with the proposed log-logistic path persistency model coupled with Poisson path birth model. This model is particularly important for indoor geolocation applications utilizing multipath diversity in the absence of DP.

\section{REFERENCES}

[1] A. A. M. Saleh and R. A. Valenzuela, "A statistical model for indoor multipath propagation," IEEE J. Select. Areas Commun., vol. 5, no. 2, Feb. 1987.

[2] R. Ganesh and K. Pahlavan, "Statistical modeling and computer simulation of indoor radio channel," IEE Proc. Commun., Speech and Vision, vol. 138, no. 3, June 1991.

[3] Thomas Zwick et al., "A stochastic multipath channel model including path directions for indoor environments," IEEE J. Select. Areas Commun., vol. 20, no. 6, Aug. 2002.

[4] Chia-Chin Chong et al., "A novel wideband dynamic directional indoor channel model based on a Markov process," IEEE Trans. Wireless Commun., vol. 4, no. 4, July 2005.

[5] K. Pahlavan et al., "Indoor geolocation in the absence of direct path," IEEE Wireless Commun. Mag., vol. 13, no. 6, pp. 50-58, Dec. 2006.

[6] B. Alavi and K. Pahlavan, "Modeling of the distance measure error using UWB indoor radio measurement," IEEE Commun. Lett., vol. 10, no. 4, pp. 275-277, Apr. 2006.

[7] D. E. Gustafson, J. M. Elwell, and J. A. Soltz, "Innovative indoor geolocation using RF multipath diversity," in Proc. IEEE/ION Position, Location, and Navigation Symposium, 2006.

[8] G. L. Turin et al., "A statistical model of urban multipath propagation," IEEE Trans. Veh. Technol., vol. 21, no. 1, pp. 1-9, Feb. 1972.

[9] A. Papoulis and S. U. Pillai, Probability, Random Variables and Stochastic Processes, 4th ed. New York: McGraw-Hill, 2002.

[10] J. F. Lawless, Statistical Models and Methods for Lifetime Data. New York: Wiley, 1982.

[11] R. C. Gupta, O. Akman, and S. Lvin, "A study of log-logistic model in survival analysis," Biometrika J., vol. 41, no. 4, pp. 431-443, Aug. 1999.

[12] T. Holt, K. Pahlavan, and J. Lee, "A graphical indoor radio channel simulator using $2 \mathrm{D}$ ray tracing," in Proc. IEEE PIMRC, Boston, USA, Oct 1992, pp. 411-416.

[13] R. Valenzuela, "A ray tracing approach to predicting indoor wireless transmission," in Proc. IEEE VTC 1993, pp. 214-218.

[14] F. O. Akgul and K. Pahlavan, "AOA assisted NLOS error mitigation for TOA-based indoor positioning systems," in Proc. IEEE MILCOM, Orlando, FL, 2007. 